\title{
Specifications and Analysis of Digitized Diagnostics of Automobiles: A Case Study of on Board Diagnostic (OBD II)
}

\author{
Medashe Michael Oluwaseyi *, Abolarin Matthew Sunday \\ Mechanical Engineering Department \\ Federal University of Technology, \\ Minna, Nigeria
}

\begin{abstract}
On-board diagnostics, (OBD) is an automotive term that refers to a vehicle's self-diagnostic and reporting ability. OBD systems enable owner of vehicles or automobile repair technician to gain access to the condition of the various vehicle sub-systems. Modern motor vehicles are highly sophisticated machines that incorporate development in electrical, electronics and mechanical engineering. The traditional "trial-and-error" mode of diagnosis could no longer be efficient to meet up with the need for maintenance and repairs on such vehicles. Therefore, this probes a challenge to meet up with the technological trend, and hence it becomes imperative to adopt new mode of diagnosis with high efficiency on the new generation motor vehicles. To facilitate and enhance the early detection of faults and malfunctions related to emissions control components, different diagnostic tools like Launch X-431 and Autel Maxidiag (Elite Series) were used and from the research carried out and the various results obtained from the various diagnostics of some selected automobiles from German Make, American Make, and Japanese Make vehicles, the diagnostics results provided an appreciated feedback through the triggering of Malfunction Indicator Light (MIL) and thereby with the use of diagnostic scan tools, the failures and faults within the engine compartment were detected. It was evident that On Board Diagnostic has the capacity to facilitate and enhance the early detection of vehicle malfunction and faults related to emissions control components and as a result reducing high emissions caused by emission related malfunctions.
\end{abstract}

Keywords-Diagnostic Trouble Code; Malfunction Indicator Light; On-board Diagnostics

\section{INTRODUCTION}

Every automobile is equipped with an electrical instrumentation panel that is used as a driver information centre, formerly known as a dashboard. It contains various gauges and indicators that provide valuable information to the driver [1]. Gauges provide scaled indication of the system condition such as distance, engine speed, vehicle speed, and fuel level. Whereas, the indicator lights supply information of something is being turned on or warn the driver about system malfunctioning problems. However, this instrumentation panel has limitation in providing more information on specific areas such as malfunctioning problems, trip information, scheduled maintenance reminder and data logging system. The information displayed on the dash board is retrieved from the electronic control unit (ECU) of the vehicle. The ECU that can provide this information normally has on-board diagnostic (OBD) software (known as a diagnostic management system).
This management system performs diagnostic testing, record the result of the tests, and request the test fail actions [2].

On-board diagnostics, (OBD) is an automotive term that refers to a vehicle's self-diagnostic and reporting ability. OBD systems enable owner of vehicles or automobile repair technician to gain access to the condition of the various vehicle sub-systems. The available diagnostic information through OBD has considerably been improved, as its introduction dates back to the early 1980s. The early versions of OBD simply illuminates a malfunction indicator light if there was any prevailing fault detected, but has no capability of further providing any information about the nature of the fault. The modern OBD systems have become more sophisticated and now adopt a standardized digital communications port to provide real time data in addition to a standardized series of diagnostic trouble codes, (DTCs), which allow one to quickly identify and repair malfunctions within the vehicle, as it provides almost complete control and also monitors parts of chassis, body and accessory devices as well as the diagnostic control network of the vehicle.

All vehicles (light and medium duty) built since January 1, 1996, in the United States, or in Asia and Europe designed to U.S specifications has On Board Diagnostics Version II (OBD II) specification, although some early OBD 2 vehicles were not $100 \%$ compliant. The OBD-II standard specifies the type of diagnostic connector and its pinout, the electrical signaling protocols available, and the messaging format. It also provides a candidate list of vehicle parameters to monitor along with how to encode the data for each. OBD-II provides access to numerous data from the engine control unit (ECU) and offers a valuable source of information when troubleshooting problems inside a vehicle.

OBD II was developed by the State of California Air Resources Board (CARB) and the United States Environmental Protection Agency (EPA) and is standardized in the US by the Society for Automotive Engineers (SAE) and worldwide (as EOBD) by the International Organization for Standardization (ISO). The SAE (J1979) standard defines a method for requesting various diagnostic data and a list of standard parameters that might be available from the ECU. The various parameters that are available are addressed by "parameter identification numbers" or PIDs which are defined in (J1979). OBD-II PIDs are codes used to request data from a vehicle, used as a diagnostic tool. All cars sold in the United States are required to use the ISO 15765-4 signaling a variant of the Controller Area Network (CAN) bus. OBD-II data can be 
collected through one of five communication protocols that are listed thus; SAE J1850 (PWM), SAE J1850 (VPW), ISO 91412, ISO 14230-4 (KWP 2000), ISO 15765-4 (CAN). The format of the request command has to follow the SAE J1979 standard (common diagnostic test mode). The OBD-II standards were primarily developed to regulate vehicles emission level standards. Many supporting systems were developed to improve the quality and performance of the instrumentation panel available in a car [3]. OBD is not a new field of study, but poor documentation, complicated interface requirements and an industry stranglehold has prevented major advancesalthough the various table text styles are provided. The formatter will need to create these components, incorporating the applicable criteria that follow.

\section{MATERIALS AND METHODS}

The materials that were used in the course of carrying out this research were the different diagnostic tools that were employed in carrying out diagnosis of fault on automobile at Sarki Pawa Automobile Workshop, along Kpakungu Gidankwano Expressway, Albishirin, Minna Niger state, Nigeria. The different diagnostic tools were Launch X431(Normal) and Autel MaxiDiag (Elite Series).

The Launch X431 auto scan tool is a new generation of product developed by Launch Tech. Available X431 products developed by Launch Tech. among others are Launch X431 (Normal), Launch X431 (Infinite) and Launch X431 (Super scanner). It is a perfect union between automotive electronic technology and information networking. X431 adopts open diagnostic technology, which is the most advanced in the world as well as the future of automotive diagnosis. It carries Personal Digital Assistant (PDA) functions apart from reading trouble code, data stream and actuation test, sensor waveform and digital code control. Important characteristics of Launch X431 include the following among others; to interface with computer with X431-PCLINK so as to store test data in vehicle service record; To simulate and test sensors with the help of sensor box; To test the parameters of the battery and conduct analysis on battery conditions through battery box; To print out test data any time with built-in printer; Easy operation through input on large touch sensitive screen; As generic diagnostic equipment, it can interface with diagnostic adaptors of all vehicles models in the world; Strong test capacity to cover all electronic systems; Unique reading of data stream to ensure fast data updating; Strong waveform display to monitor any change in the data stream; Embedded operation system and open-platform diagnosis to make effective diagnosis possible; Integrated adaptors featuring compact design and reliability; Multi-language choices and easy update through the Internet.

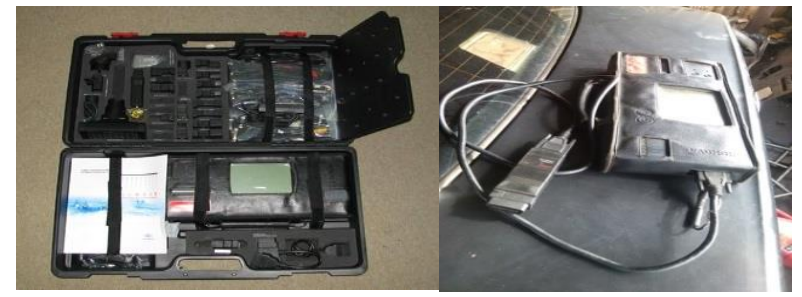

Figure 1: Complete package of a Launch X431 (Normal) with adapters for connectors of several vehicle manufacturers.
Autel scan tools are powerful and professional auto tools for diagnosis. Autel Scan tools have many different series including maxidiag, maxidas, autolink and more. Autel new MaxiDiag Elite Series, is the most advanced and multifunctional scan tools powered by its exclusive technology and designed for technicians to troubleshoot either the basic four systems or all the systems for most of the major vehicles on the road today.

\section{Method/Procedure in Carrying out Diagnosis}

The data processed in this report were all gather at Sarki Pawa Automobile Workshop along Kpakungu- Gidankwano expressway Minna, Niger state, Nigeria during the period of this research for different make and model of automobiles. The information obtained includes the following; model year (MY), make, model, Engine Number, and the Diagnostic Trouble Codes (DTC) for all the automobiles covered in this report.

Although there are generalised procedures of carrying out diagnostics, but individual diagnostic tool has their specific procedures of carrying out their diagnostics.

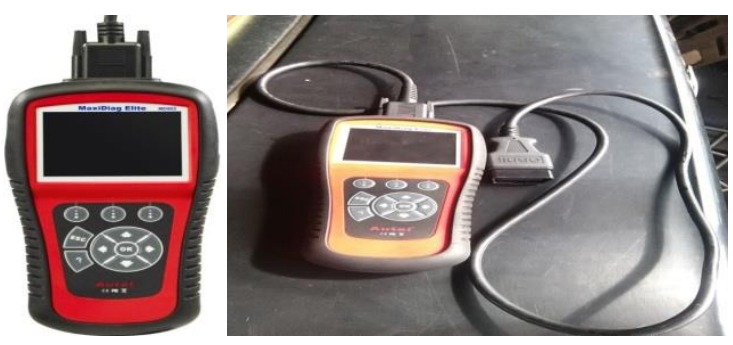

Figure 2a: Autel Scan Tool (MaxiDiag Elite Series)

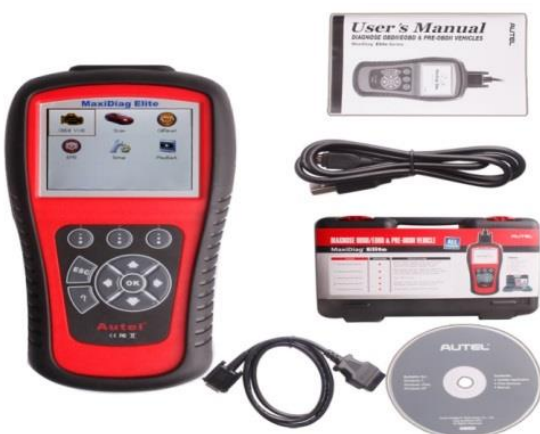

Figure 2b: Complete package of Autel Scan Tool (MaxiDiag Elite Series) Scan tool, DLC, DVD, Carry Case, USB Cable, and user manual.

\section{Diagnostic with Launch $X-431$}

To carry out diagnostic with X-431, the following are the step by step procedure to be undertaken;

There are a few things to keep in mind when using the X-431.

i. Always use the OEM connector that comes with the $\mathrm{X}-431$, even if the vehicle has an OBDII socket.

ii. If the unit is cold, the monitor may not function properly. Allow it to warm up before using.

iii. The X-431 is powered by the vehicle's battery. If the battery voltage is less than 10 , the unit will notify you by beeping. 
By now the Scan tool is ready to be connected to the car. The following are the steps to be followed;

1. Take the appropriate connector and attach it to the free end of the main cable. Then take that end and attach it to the car.

2. Press the On/Off button to turn the X-431 on.

3. Allow it to run until you see "LAUNCH" displayed across the screen.

Then press the Hot Key (button above the power key). The following screen will appear:

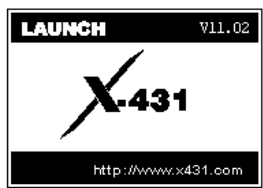

Start $4:$ 10:34
Figure 3: Startup screen of $X-431$

4. Touch START. A screen resembling the following will be displayed for the vehicle make to be selected.

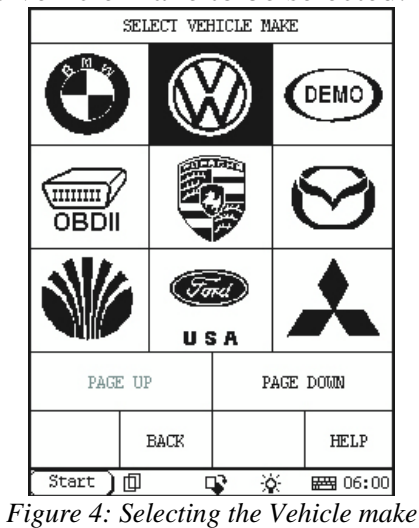

5. Select the car line to which you are connected (Volkswagen was selected). From this point on, the X-431 mirrors the OEM factory tool.

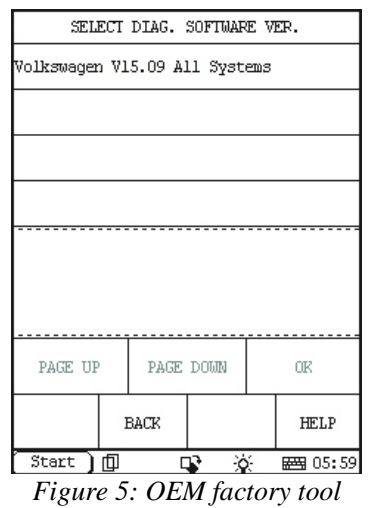

6. Choose the software version that you would like to use to scan the vehicle.

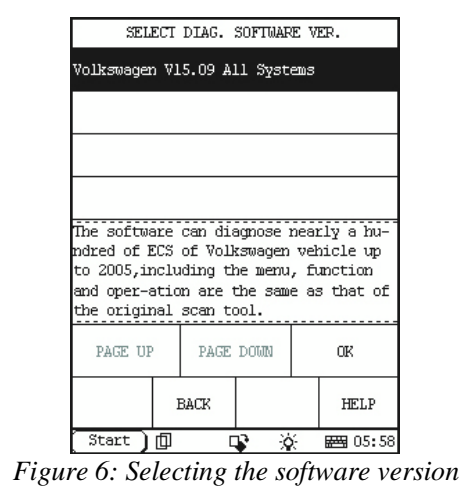

The latest version will be listed on top. Select it and then press OK.

7. The Smart box will now be initialized (that is, the software stored on the

CF Card will be uploaded to the Smart box), as shown below.

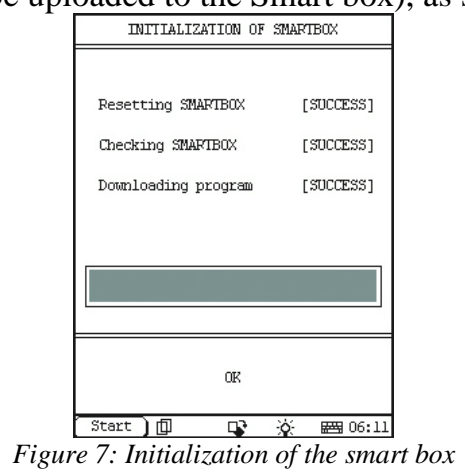

When initialization is finished, press $\mathbf{O K}$.

8. Launch $\mathrm{X}-431$ is now ready for diagnosis. A screen similar to the following is displayed. (It is worthy to note, for some vehicles lines, you may be required to select the vehicle model on which you are working before this screen is displayed.)

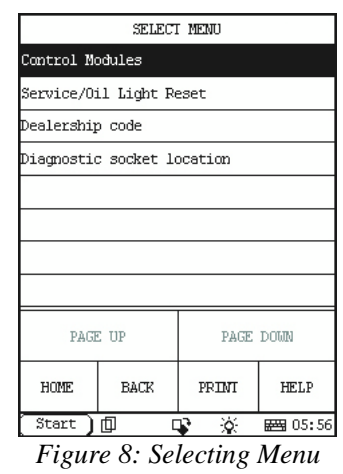

At this point, the X-431 scan tool will become highly intuitive and is really very user friendly. Then from the systems displayed, it can be chosen to find out which systems have codes.

\section{Diagnostics with Autel (MaxiDiag Elite Series)} Location of the Data Link Connector (DLC)

The DLC (Data Link Connector or Diagnostic Link Connector) is the standardized 16-cavity connector where diagnostic scan tools interface with the vehicle's on-board PCM. The DLC is usually located from the center of the instrument panel (dashboard), under or around the driver's side for most vehicles. If Data Link Connector is not located 
under dashboard, a label will be there telling the location. If the DLC cannot be found, refer to the vehicle's service manual for the location.

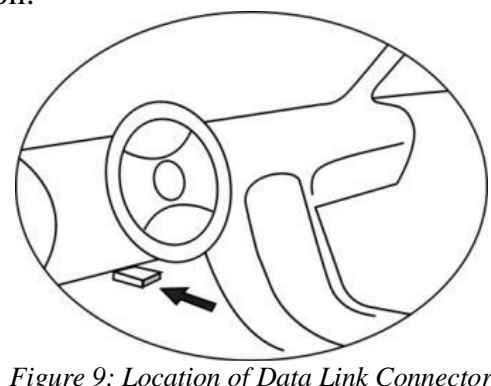

\section{Cable connection to vehicle}

During vehicle testing, power for the scan tool is usually provided through the vehicle cable connection. When the scan tool is not connected to a vehicle, the scan tool can be powered with an AC/DC external power adapter.

While the scan tool is powered via the vehicle Data Link Connector (DLC), the following steps can be followed to turn on the scan tool:

1. Connect the Cable to scan tool.

2. Find DLC on vehicle.

Note: For some vehicles, it may be required to remove a plastic DLC cover before plugging the OBD2 cable.

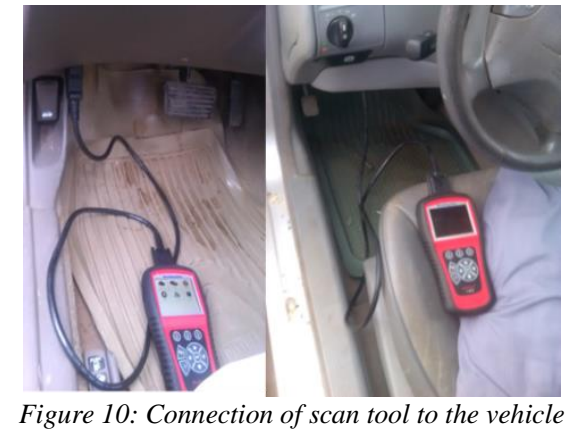

3. Plug the cable to the vehicle's DLC.

4. Power up the scan tool, and wait for the Main Screen to appear. (Figure 3.19)

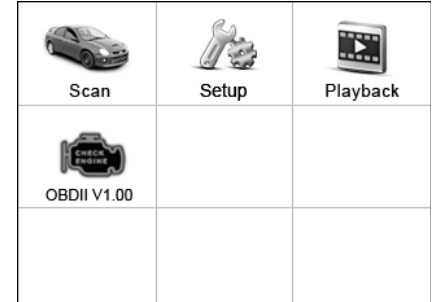

Figure 11: Main Screen of Autel Scan when power up

\section{Entering vehicle information}

Before using the scan tool to diagnose, it is required to input the vehicle information. There are generally three ways to input the vehicle information.

i. Vehicle information manual acquisition.

ii. VIN code automatic acquisition.

iii. VIN code manual acquisition.
The way to enter diagnostic procedure depends on vehicle being tested.

\section{Vehicle information manual acquisition}
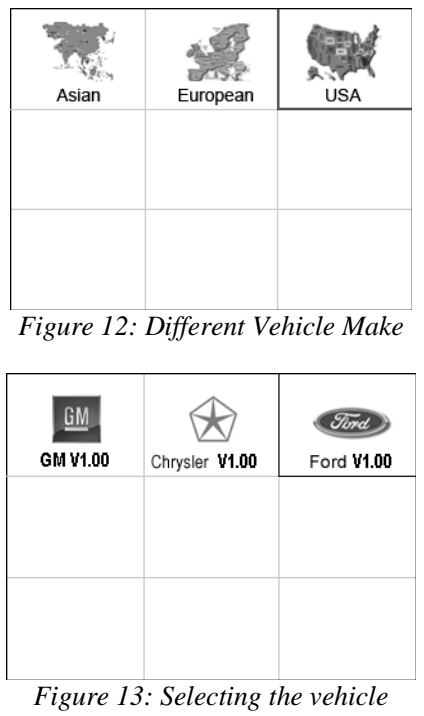

The following steps are to be followed to enter the vehicle information and begin diagnostics. (Taking Ford as an example)

1. Turn on the scan tool and wait for the Main Screen to appear.

2. Select Scan icon in the Main Screen (Figure 3.20) and wait for the vehicle manufacturer screen. Choose the correct vehicle make.

3. Step by step, select the right options for your vehicle according to each screen that appears.

4. Do this until the complete vehicle information is entered. Then the scan tool will ask your confirmation.

VIN code automatic acquisition

Some vehicles could identify the VIN code intelligently, saving the technician time to input complex information. (Taking Renault as an example)

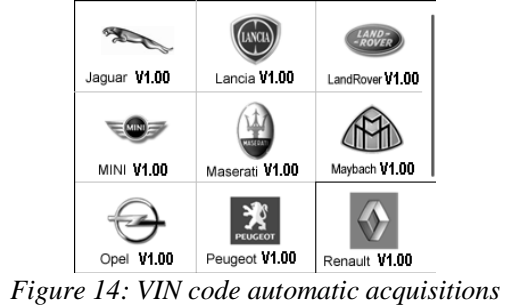

In this mode, the scan tool will communicate with the vehicle and read off the VIN code automatically, and then it will ask for your confirmation if the VIN code is correct. If the VIN code is incorrect, it will turn to manual mode to input VIN code.

\section{VIN code manual acquisition}

For some vehicles, both selecting the options manually and acquiring the VIN are available for you to enter the vehicle information. (Taking Benz as an example) 


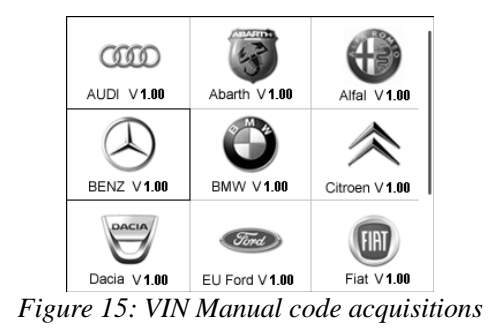

In the Benz Cars menu, choose the item "2". Select by entering VIN" and you can enter the VIN code directly.

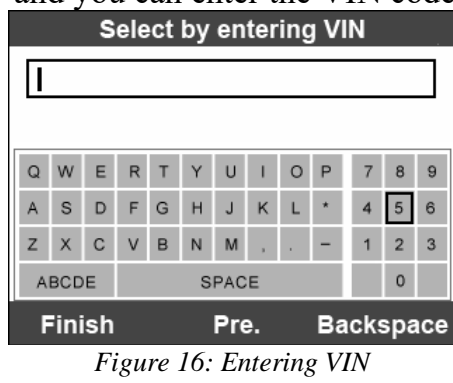

When you choose to enter VIN directly, a pop-up soft keyboard is used to input VIN code. (Figure 3.24)

To pop up the keyboard, press the Function button corresponding to Show. Use UP/DOWN scroll button and LEFT/RIGHT scroll button to select digit and character, and then press $\mathbf{O K}$ button to confirm. Use Backspace button to delete the previous digit or character. When finished, press the Function button corresponding to Finish to proceed. The scan tool will identify the VIN code and turn to diagnostic procedure.

\section{Diagnostic Test}

After the correct vehicle information has been entered, the diagnostic testing selection will display:

\section{Auto Scan}

Depending on the scan tool model, Auto Scan function will carry out an overall scan to check the status of all systems or four systems (engine, transmission, airbag and ABS) on the vehicle being tested. Selecting Auto Scan will lead to retrieve the trouble codes in each system of the vehicle one by one. It will take a few minutes to display.

Use the UP/DOWN scroll button to select Auto Scan from Select an Option menu, and press the OK button.

The User is allowed to check the details of each system, quickly erase DTC, save the data, and display DTC from the Auto Scan menu screen. To select the options on the bottom, simply press the corresponding function button.

Save: - You can save the Auto Scan information as "Vehicle Record" so that you will not need to follow the vehicle selection process again on the same vehicle in later tests.

Quick Erase: - By selecting this option, the scan tool will erase all displaying DTCs and once again read the data and check the latest status of the system. If the system did not repair, the trouble codes will keep on displaying.

Display DTC: - This option allows you to read DTC definitions in the highlighted system. If more than one fault is detected in a system, the scan tool will display an option list to view different kind of DTCs or freeze frames.

In Auto Scan screen, pressing OK button will turn to diagnostic operation.

\section{Diagnostic Operation}

This function allows you to read and clear diagnostic trouble codes (DTCs) from a vehicle.

\section{Read Codes}

The Read Codes procedure varies for each vehicle being tested. This section includes the following Read Codes procedures.

In the Function Menu screen, select Read Codes. This will display the Read Codes menu screen.

In the Read Codes menu, select one of the options to proceed. Select one of the DTC options to view detailed diagnostic trouble code information.

You can save the code results for later review by selecting Save option on the bottom. When you finished viewing the DTCs, press the ESC button to return to previous screen.

\section{Erase Codes}

After reading and / or reviewing the diagnostic trouble codes, use the following steps to erase the codes from the vehicle. If Erase Codes is not an available menu option, consult the manufacturer's service manual for the correct "clear code" method. This Erase Codes function clears the DTCs from the selected ECU or provides instructions for how to manually clear the codes from the ECU. Before performing this procedure, it is important to ensure the vehicle's ignition key is in the On (Run) position with the engine off. (KOEO)

\section{To Erase DTCs, the following steps are undertaken:}

1. With the Function Menu screen displayed, click on Erase Codes. The scan tool displays an instruction message.

2. Follow the instructions on each screen that appears until the procedure is complete.

3. When finished, press any key to exit.

4. Use Read Codes function to check the codes again to see if DTCs have been erased successfully. If any codes remain, repeat the Erase Codes steps.

\section{RESULTS \& DISCUSSION}

The vehicle information (Make, Model Year, and Engine) and Diagnostics Trouble Codes (DTCs) used for the analysis carried out in this report were as collected from actual diagnosis of Customer's Vehicles at Sarki Pawa Automobile Workshop. The data collected covers some selected Japanese, German and American vehicles from 2001 Model Year to 2011 Model Year. 
Table 1: Vehicle Make, Model Year, Engine and DTCs for some selected German Vehicles

\begin{tabular}{|c|c|c|c|c|}
\hline Vehicle Make & Model & Model Year & Engine & DTCs \\
\hline Volvo & S60 T5 & 2001 & L5-2.3L Turbo VIN53B5234T3 & P1171 \\
\hline Mercedez Benz & E320 Sedan (210.065) & 2002 & V6-3.2L (112.941) & P0011 \\
\hline Volvo & S80 T6 Executive & 2003 & L6-2.9L Turbo VIN91B6294T & P0300 \\
\hline Mercedez Benz & E320 4MATIC Wagon (211.282) & 2003 & V6-3.2L (112.954) & P0156 \\
\hline Mercedez Benz & E320 Sedan (211.065) & 2003 & V6-3.2L (112.949) & P0171 \\
\hline BMW & 325i Sedan (E46) & 2005 & L6-2.5L (M56) & P1182 \\
\hline Volkswagen & Passat Sedan & 2008 & L4-2.0L Turbo & P0056 \\
\hline Mercedez Benz Truck & GL450 (164.871) & 2008 & V8-4.6L(273.923) & P0306 \\
\hline Mercedez Benz & C300 4MATIC (204.081) & 2008 & V6-3.0L (272.948) & P0154 \\
\hline Volkswagen & Passat Sedan & 2010 & L4-2.0L Turbo (CCTA) & P0410 \\
\hline Volkswagen & Passat Sedan & 2011 & L4-2.0L Turbo & P0431 \\
\hline
\end{tabular}

Table 2: Vehicle Make, Model Year, Engine and DTCs for some selected American Vehicles

\begin{tabular}{|c|c|c|c|c|}
\hline Vehicle Make & Model & Model Year & Engine & DTCs \\
\hline Jeep Truck & Cherokee 4WD LHD & 2001 & L6-4.0L VIN S & P0075 \\
\hline Ford Truck & Ranger 2WD & 2001 & V6-4.0L VIN E & P0102 \\
\hline Ford Truck & Explorer 4WD & 2002 & V6-4.0L VIN E & P0118 \\
\hline Ford Truck & Explorer 4WD & 2003 & V8-4.6L VIN W & P1651 \\
\hline Ford Truck & Explorer 4WD & 2004 & V8-4.6L SOHC VIN W & P0080 \\
\hline Ford & Five Hundred AWD & 2006 & V6-3.0LVIN 1 & P1451 \\
\hline Ford & Fusion & 2006 & V6-3.0L VIN 1 & P1650 \\
\hline Jeep Truck & Liberty 4WD & 2008 & V6-3.7L & P0506 \\
\hline GMC Truck & Envoy 4WD & 2009 & L6-4.2L & P0171 \\
\hline Chevrolet & Corvette & 2010 & V8-7.0L & P1860 \\
\hline Ford Truck & F 250 2WD Super Duty & 2011 & V10-6.8L & P0102 \\
\hline
\end{tabular}

Table 3: Vehicle Make, Model Year, Engine and DTCs for some selected Japanese Vehicles

\begin{tabular}{|c|c|c|c|}
\hline Vehicle Make & Model & Model Year & Engine \\
\hline Toyota Truck & Highlander4WD & 2002 & V6-3.0L (1MZ-FE) \\
\hline Toyota & Camry LE Sedan & 2002 & L4-2.4L (2AZ-FE) \\
\hline Nissan Datsun & Sentra XE & 2002 & L4-1.8L (QG18DE) \\
\hline Honda & Civic LX Sedan & 2002 & P1135 \\
\hline Mazda Truck & MPV & 2003 & P0011 \\
\hline Toyota & Corolla CE Sedan & 2003 & P6-3.0L DOHC \\
\hline Toyota Truck & Highlander 4WD & 2004 & L4-1.8L (1ZZ-FE) \\
\hline Honda & Civic & 2007 & V6-3.3L (3MZ-FE) \\
\hline Honda & Civic Si & 2007 & L4-1.8L \\
\hline Toyota Truck & Highlander4WD & P0304 & L4-2.0L \\
\hline Honda & Civic & 2008 & P0420 \\
\hline
\end{tabular}

\section{Discussion of Results}

The experimental results (DTCs) obtained from the various diagnostic carried out on some selected vehicle Make, MY are thus, analyzed as follows;

\section{Analysis of DTCs from Selected German Vehicles P1171: Long Term Fuel Trim}

The control module receives information from the heated oxygen sensor $\left(\mathrm{H}^{\mathrm{O}_{2}} \mathrm{~S}\right)$ about the fuel-air mixture during the idle cycle at both the lower and upper part-load range. When the short-term fuel trim makes an adjustment, the integrator median must be adjusted by the long-term trim. Diagnostic trouble code (DTC) ECM-261A will be stored when the longterm trim must be adjusted to almost maximum in the lower part load area.

\section{Possible Cause}

Upper limit:

$>$ Leakage of intake air

$>$ leakage of exhaust system air

$>$ low fuel pressure

$>$ heated oxygen sensor $\left(\mathrm{H}^{O_{2} \mathrm{~S}}\right)$ is defective

Lower limit:

$>$ leakage of intake air

$>$ high fuel pressure

$>$ leakages of injectors

$>$ defective mass air flow (MAF) sensor

$>$ contaminated engine oil

$>$ oil level too high

$>$ defective heated oxygen sensor $\left(\mathrm{H}^{\mathrm{O}_{2} \mathrm{~S}}\right)$ 
P0011: "A" Camshaft Position - Timing Over-Advanced or System Performance (Bank 1)

A code P0011 refers to the VVT (variable valve timing) or VCT (variable camshaft timing) components and the car's PCM (powertrain control module, also called an ECM). That consists of a few different components but the P0011 DTC specifically refers to the camshaft (cam) timing. In this case, if the cam timing is above a set limit (over-advanced), the Check Engine Light will be illuminated and the code will be set. The "A" camshaft is either the intake, left, or front camshaft.

Potential Symptoms

Most likely a P0011 DTC will result in one of the following: hard starting, poor idle, and/or stalling. There are potentially other symptoms as well. Of course, when trouble codes are set, the MIL (malfunction indicator lamp, a.k.a. the check engine light) illuminates.

\section{Causes}

A P0011 DTC trouble code may be caused by one or more of the following:

$>$ Incorrect camshaft timing

$>$ Wiring problems (harness/wiring) in intake timing control valve control solenoid system

$>$ Continuous oil flow to VCT piston chamber

$>$ Failed timing valve control solenoid (stuck open)

\section{Possible Solutions}

This DTC code is a result of a mechanical fault of the VCT unit or related components, so there is no need for electrical diagnosis.

P0300 - Random/Multiple Cylinder Misfire Detected

Basically this means that the car's computer has detected that not all of the engine's cylinders are firing properly. A P0300 diagnostic code indicates a random or multiple misfire. If the last digit is a number other than zero, it corresponds to the cylinder number that is misfiring. A P0301 code, for example, would tell you cylinder number one is misfiring. Unfortunately, the limitation of a P0300 is that it does not tell one specifically which cylinder(s) is/are misfiring, nor why.

\section{Symptoms}

Symptoms may include:

$>$ the engine may be harder to start

$>$ the engine may stumble, and/or hesitate

$>$ other symptoms may also be present like illumination of MIL

\section{Possible Causes}

A P0300 code may mean that one or more of the following has happened:

$>$ Faulty spark plugs or wires

$>$ Faulty coil (pack)

$>$ Faulty oxygen sensor(s)

$>$ Faulty fuel injector(s)

$>$ Burned exhaust valve

$>$ Faulty catalytic converter(s)

$>$ Stuck/blocked/leaking EGR valve or passages

$>$ Faulty camshaft position sensor

\section{Possible Solution}

If there are no symptoms, the simplest thing to do is to reset or erase the code and see if it comes back.

If there are symptoms such as the engine is stumbling or hesitating, check all wiring and connectors that lead to the cylinders (i.e. spark plugs). Depending on how long the ignition components have been in the car, it may be a good idea to replace them as part of regular maintenance schedule. I would suggest spark plugs, spark plug wires, distributor cap, and rotor (if applicable). Otherwise, check the coils (a.k.a. coil packs). In some cases, the catalytic converter has gone bad. If there is smell of rotten eggs in the exhaust, the cat converter needs to be replaced.

\section{P0156: Oxygen $\left(\mathrm{O}_{2}\right)$ Sensor Circuit Malfunction (Bank 2 Sensor 2)}

The $\mathrm{O}_{2}$ (oxygen) sensors measure the amount of oxygen content in the exhaust. The PCM (powertrain control module) then uses this information to regulate fuel injector pulse. The $\mathrm{O}_{2}$ sensors are very important for the proper operation of the engine. Inaccurate or faulty $\mathrm{O}_{2}$ sensors can cause the PCM to add or take away fuel based on the faulty $\mathrm{O}_{2}$ sensor voltage which can cause a host of problems. Also for the $\mathrm{O}_{2}$ sensor heater element there is a battery voltage supply wire and another ground circuit for that. The $\mathrm{O}_{2}$ sensor heater allows the $\mathrm{O}_{2}$ sensor to warm up faster, thus achieving closed loop in less time than it would normally take for the exhaust to warm the sensor up to operating temperature. The $\mathrm{O}_{2}$ sensor varies the supplied reference voltage based on oxygen content in the exhaust.

\section{Symptoms}

Although $\mathrm{O}_{2}$ do not directly control fuel, often times $\mathrm{O}_{2}$ sensor problems present few symptoms since they are inputs to the PCM to monitor catalytic converter quality. Sometimes no symptoms are noticeable. However the following may be possible on some vehicles:

$>$ MIL (Malfunction Indicator Lamp) illumination

Causes

Increase in tailpipe emissions

Potential causes of a P0156 code include the following:

$>\mathrm{Bad}_{2} \mathrm{O}_{2}$ sensor Signal shorted to voltage

$>$ Wiring problems due to contact with exhaust components

$>$ Holes in exhaust near $\mathrm{O}_{2}$ sensor

\section{Possible Solutions}

A scan tool can be used to check the signal voltage for the Bank 2, 2 oxygen sensor with the engine at normal operating temperature. Is it stuck low currently? If so, increase RPM for a few seconds and see if it affects the reading. If it begins working with increased RPM, check for holes in the exhaust near the $\mathrm{O}_{2}$ sensor that may cause a false lean. If the exhaust pipe is intact, this then imply that sensor is sluggish and hence should be replaceed. If the Bank 2, $2 \quad \mathrm{O}_{2}$ sensor voltage reading remains low with increased RPM, unplug it and then observe the reading. If it did, check for water intrusion or 
other connector problems. If none is found, replace the shorted $\mathrm{O}_{2}$ sensor. checks out, suspect the PCM.

\section{P0171- Fuel Trim System Too Lean}

The adaptive fuel strategy in the vehicle's computer constantly monitors the fuel delivery system to make sure the engine is running at an optimum air to fuel ratio, which is $14.7: 1$ (15:1 approximately). The computer adjusts the injector pulse width to regulate amount of fuel going into the engine. The oxygen sensors relay information to the powertrain Control Module, informing it of the content in the exhaust. This information is translated by the computer, and used to determine whether more or less fuel is needed. The computer will then adjust fuel flow, to keep the correct air fuel mixture. The loop continues as long as the engine is running. A P0171 trouble code tells you the fuel mixture is running lean (not enough fuel and/or too much air). This code triggered the Check Engine Light when the computer has reached a rich calibration and cannot add enough fuel to maintain the correct mixture.

\section{Possible Causes and Remedy}

\section{A lean fuel condition can be caused by:}

$>$ Low fuel pressure due to a weak pump or leaky fuel pressure regulator. A fuel pressure gauge can be used to check fuel pressure at idle cycle. If fuel pressure is less than specifications, your fuel filter may be plugged, your fuel pump may be failing or have a bad wiring connection, or the fuel pressure regulator may be leaking.

$>$ Dirty fuel injectors. Cleaning the injectors with a fuel system additive, or having the injectors professionally cleaned may solve the problem.

$>$ Vacuum leaks at the intake manifold, vacuum hose connections or throttle body.

$>$ Leaky EGR valve. Check the operation of EGR valve and system, and for a buildup of carbon under the valve.

$>$ Leaky PCV Valve or hose. (Check valve and hose connections)

$>$ Dirty or defective Mass Airflow Sensor (MAF). Try cleaning the MAF sensor wires or filament with aerosol electronics cleaner. Do NOT use anything else to clean the sensor, and do not touch the sensor wires.

P1182: $\mathrm{O}_{2}$ Sensor (Bank 1 Sensor 2) Open Circuit during Coast down Fuel Cut-off

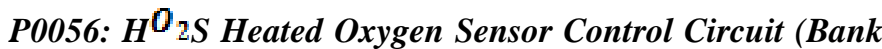 2 Sensor 2)}

Fuel injected vehicles use heated oxygen sensors in the exhaust system before and after the catalytic converters to determine oxygen content. This feedback is used to adjust the fuel system accordingly to maintain a proper air-fuel ratio of 14.7:1 (approximately 15:1). The oxygen sensors used a heated circuit to warm up the sensor for faster feedback operation. The oxygen sensor may use three or four wires depending on the vehicle, two are usually used for the sensor feedback to the Powertrain Control Module (PCM) or Engine Control Module (ECM) and the other wires are for the heater to power the heated circuit. Three wire sensors are usually grounded through the exhaust system, and four wire sensors have a separate ground wire. The P0056 code refers to the sensor after the catalytic converter on Bank 2, which is on the side of the engine that does NOT contain the number 1 cylinder. The heater circuit may be supplied power or ground by the PCM/ECM or another source that can be controlled by the PCM/ECM. It is important to note that this code is similar to P0030 and basically identical to P0036.

\section{Symptoms}

Symptoms of a P0056 DTC includes; MIL (Malfunction Indicator Light) illumination. You will probably not notice any other symptoms associated with the failure of the heated circuit since it only runs momentarily when the vehicle is first started. This sensor is also after the catalytic converter, so it will not affect the air-fuel ratio input to the PCM/ECM; it is primarily used to verify the catalytic converters efficiency.

Causes

Potential causes of a P0056 trouble code may include: Open circuit inside oxygen sensor or open power or ground wires to oxygen sensor Exhaust system ground strap may have become corroded or broken PCM/ECM or oxygen sensor heater circuit wiring has failed

Possible Solutions

Visually inspect the oxygen sensor wiring for damaged or loose wiring to the sensor. Unplug the oxygen sensor and using a digital volt ohm meter (DVOM) set to the ohms scale, test the resistance of the heater circuit using a wiring diagram for reference. The heater circuit inside the sensor should have some resistance present, excessive resistance or an over limit reading would indicate an open in the heated portion of the circuit and the oxygen sensor will need to be replaced. Backprobe the ground wire at the connector and check for resistance between a good known ground and the connector to the oxygen sensor. Back-probe the power supply wire at the connector with the DVOM set to DC volts with the positive lead on the power supply wire and the negative lead at a good known ground to check for power to supply at the oxygen sensor. If no power is present at the connector during initial car startup (cold start), there may be a problem with the power supply circuit to the oxygen sensor or the PCM itself.

\section{P0306: Cylinder Number 6 Misfire Detected}

A P0306 code means that the car's computer has detected that one of the engine's cylinders is not firing properly. In this case it's cylinder Number 6 .

Symptoms

Symptoms may include:

$>$ the engine may be harder to start

$>$ the engine may stumble, and/or hesitate

$>$ other symptoms may also be present

\section{Causes}

A code P0306 may mean that one or more of the following has happened:

$>$ Faulty spark plug or wire

$>$ Faulty coil (pack)

$>$ Faulty oxygen sensor(s)

$>$ Faulty fuel injector 
$>$ Burned exhaust valve

$>$ Faulty catalytic converter(s)

$>$ Running out of fuel

$>$ Poor compression

$>$ Defective computer

\section{Possible Solutions}

If there are no symptoms, the simplest thing to do is to reset or erase the code and see if it comes back. If there are symptoms such as the engine is stumbling or hesitating, check all wiring and connectors that lead to the cylinders (i.e. spark plugs). Depending on how long the ignition components have been in the car, it may be a good idea to replace them as part of your regular maintenance schedule. I would suggest spark plugs, spark plug wires, distributor cap, and rotor (if applicable). Otherwise, check the coils (coil packs). In some cases, the catalytic converter has gone bad. If there is a smell of rotten eggs in the exhaust, it imply the cat converter needs to be replaced. This could in some cases be problems of faulty fuel injectors.

\section{P0154: Oxygen $\left(\mathrm{O}_{2}\right)$ Sensor No Activity Detected (Bank 2, Sensor 1)}

The oxygen sensors are critical to the engine running properly. It basically informs the PCM (Powertrain Control Module) of oxygen content of the exhaust. The PCM then uses this information to regulate fuel into the engine and maintain proper air: fuel ratio. It is a four wire sensor, with the PCM providing a reference/signal voltage of about half a volt $(0.5 \mathrm{v})$ to the sensor. The other two wires are dedicated to the oxygen sensor heater element. This heater allows the sensor to warm up faster, which allows the engine to enter closed loop faster, reducing startup emissions. The heater element is supplied a $12 \mathrm{v}$ feed from the power distribution center (usually) and a ground. The oxygen content of the exhaust affects the $\mathrm{O}_{2}$ sensor resistance. This resistance produces a counter voltage on the reference/signal wire that the PCM will use to analyze oxygen in the exhaust. Lean exhaust produces low voltage, while rich exhaust produces high voltage. If for some reason the $\mathrm{O}_{2}$ sensor doesn't switch properly or "sticks", P0154 may set. This code indicates the $\mathrm{O}_{2}$ sensor is not operating.

\section{Symptoms}

Symptoms of a P0154 code may include:

$>$ MIL (Malfunction Indicator Lamp) illumination

$>$ Other codes indicating rich or lean condition may be present

$>$ Poor idle, won't idle

$>$ Misfire at idle or at highway speed

$>$ Engine may blow black smoke at tailpipe

$>$ Fuel economy may decrease

$>$ May start and stall

\section{Causes}

Potential causes of a P0154 code include:

$>$ Faulty bank 2,1 Oxygen sensor

$>$ Holes in exhaust near oxygen sensor

$>$ Short to voltage or ground on signal circuit

$>$ Open or high resistance in signal circuit
$>$ Wiring harness chafing/rubbing on exhaust components

$>$ Water/oil intrusion at $\mathrm{O}_{2}$ sensor connector

$>$ Broken lock or loose terminals on $\mathrm{O}_{2}$ sensor connector

$>$ Oil/coolant fouled oxygen sensor

\section{Possible Solutions}

First start the engine and bring it up to operating temperature and ensure the engine reaches closed loop. Then, using the live data function on a scan tool, observe the Bank 2, 1 oxygen sensor voltage reading. Is it switching properly? If so, the problem may be an intermittently bad sensor or more likely a wiring problem. Visually check the $\mathrm{O}_{2}$ sensor wiring and repair as needed. Does the voltage for the Bank 2, 1 oxygen sensor appear to be "stuck" and not moving? If so, increase idle speed for 30 seconds or so. If the sensor begins switching after a period of elevated idle, visually check the exhaust for holes or rust near the $\mathrm{O}_{2}$ sensor that could be affecting the voltage reading if the exhaust checks out, suspect the sensor to be sluggish and replace it. If the Bank 2, 1 oxygen sensor appears to be not switching, turn the engine off, and unplug the Bank 2, 1 oxygen sensor. With KOEO (Key on engine off) jumper the $\mathrm{O}_{2}$ sensor signal wire to the ground wire. Now the voltage reading should be low (about $0.1 \mathrm{v}$ ). If it is, then check for a bad connection at the $\mathrm{O}_{2}$ sensor connector. Repair as necessary. If no bad connection is found, replace the $\mathrm{O}_{2}$ sensor and re-check. If when you jumper the signal wire to the ground wire the voltage reading isn't low (about $0.1 \mathrm{v}$ ), remove the jumper wire. Now check for voltage at the $\mathrm{O}_{2}$ sensor signal wire. It should have, with KOEO and $O_{2}$ sensor unplugged, roughly 0.5 volts. If it does, check also for a good ground to the sensor as well. Repair as necessary Check for loose connections, water intrusion at PCM connector, etc. If you have no 0.5 volt reference voltage, unplug the PCM connector and ohm the signal circuit and ground circuits. There should be no resistance or any voltage. Repair excessive resistance. If you still have no 0.5 volt reference voltage, recheck at the PCM connector. It may be necessary to clip the signal wire to eliminate the possibility of a short or open somewhere. If you now have reference voltage present coming out of the PCM, fix open/short in signal circuit. If you have no reference voltage coming out of the PCM, the PCM will have to be diagnosed for a fault. It may be the problem.

\section{P0410: Secondary Air Injection (AIR) Malfunction}

The most common trouble codes or P Codes associated with broken or cracked vacuum hoses are: P0410 Secondary air injection (AIR) system malfunction, P0411 Secondary air injection (AIR) system incorrect flow detected or P1423 Secondary air injection (AIR) system throughput too small. This straightforward fix will require VW P/N: 1JM 133001 vacuum hose. Vacuum hose material changed with introduction of engine codes $\mathrm{AVH}$ and $\mathrm{AZG}$. The engine number code letters and serial number can be found on the front of the engine near the engine / transmission joint. The engine/serial number may also be located on a label on the cylinder head cover and vehicle data plate. 


\section{P0431: "Warm up catalyst efficiency below threshold (bank} 2)"

Basically this means that the oxygen sensor downstream of the catalytic converter on bank 2 is detecting that the converter is not working as efficiently as it should be (according to specifications). It is part of the vehicle emissions system.

Symptoms

You will likely not notice any drivability problems, although there may be symptoms such as a rough/hard idle when cold.

\section{Causes}

A code P0431 may mean that one or more of the following has happened:

$>$ The catalytic converter is no longer functioning properly

$>$ An oxygen sensor is not reading (functioning) properly

$>$ There is an exhaust leak

\section{Possible Solutions}

Inspect for exhaust leaks. Next step is to measure the voltage at the oxygen sensor on Bank 2. In fact, it would be a good idea to test each oxygen ${ }^{O_{2}}$ sensor while you're at it. Analysis of DTCs from Selected American Vehicles

\section{P0075: Intake Valve Control Solenoid Circuit Bank 1}

Intake Valve Control Solenoid Circuit Bank 1 is the generic definition for the P0075; however different vehicle's manufacturer may have a different definition and information for the P0075 code.

Possible symptoms

- Engine Light ON (or Service Engine Soon Warning Light)

Possible Causes

$>$ Faulty Intake Valve Control Solenoid Bank 1

$>$ Intake Valve Control Solenoid Circuit Bank 1 harness is open or shorted

$>$ Intake Valve Control Solenoid Circuit Bank 1 circuit poor electrical connection

\section{P0102: Mass Air Flow (MAF) Circuit Low Input}

Basically this means that there is a problem with the Mass Air Flow (MAF) sensor or circuit. A more technical description would be that the MAF circuit had lower than expected voltage (air flow). Other MAF sensor circuit DTC trouble codes are P0100, P0101, P0103, and P0104.

\section{Symptoms}

You will likely not notice any serious drivability problems, although there may be symptoms such as a general decrease in power or sluggishness.

Causes

A code P0102 may mean that one or more of the following has happened:

$>$ The MAF may be disconnected, or a wiring connection may be bad

$>$ The MAF may be dirty or otherwise contaminated (Note: if you use a reusable oiled air filter, be careful not to apply too much oil or that can contaminate the MAF).

$>$ The MAF sensor may be faulty

\section{Possible Solutions}

The simplest thing to do is to reset or erase the code and see if it comes back. Then start with the cheapest, easiest repair procedures: Verify that the Mass Air Flow Sensor wiring is connected properly and that there are no broken / frayed wires. Inspect for any air leaks near the MAF sensor. Take the MAF out and clean it using a spray cleaner such as brake cleaner or electrical contact cleaner. Be gentle with the sensor. Replace the MAF sensor.

\section{P0118 - Engine Coolant Temperature Circuit High Input}

The engine coolant temperature (ECT) sensor is a thermostat screwed into a coolant passage in the cylinder head. Sensor resistance is high when coolant temperature is low and resistance drops when coolant temperature increases. The powertrain control module (PCM) provides a 5 volt reference and a ground to the sensor. The PCM monitors voltage drop to determine coolant temperature. If the ECT reads less than freezing temperature, when engine has been running for more than a few minutes, the PCM determines a circuit fault and sets this code. Or if the PCM determines the sensor resistance is out of specifications, this code is set.

\section{Potential Symptoms}

Symptoms of a P0118 could include:

$>$ Very poor fuel economy

$>$ A no start condition

$>$ Vehicle may start, but run very poorly, blowing black smoke, running very rough and misfiring

Causes

$>$ Illumination of MIL

A code P0118 may mean that one or more of the following has happened:

$>$ A bad connection at the sensor

$>$ An open in the ground circuit between the ECT sensor and the PCM

$>$ A short in the voltage feed between the sensor and the PCM

$>$ A bad PCM (less likely)

$>$ A bad temperature sensor (shorted internally)

\section{Possible Solutions}

First, with the help of a scan tool, check the reading of the coolant sensor. Does it read a logical number? If so, the problem is likely intermittent. Perform a "wiggle" test by wiggling the connector and harness to the sensor while watching the reading on the scan tool. Watch for any dropouts. Drop-outs would indicate a bad connection. If the scan tool reads an illogical temperature, check the resistance of the temperature sensor. If it is out of specifications, replace it. If it is in specifications, unplug the sensor and, using a fused jumper wire, jumper the two terminals of the connector together. The temperature reading should now be maxed out to above 250 degrees $\mathrm{F}\left(121^{\circ} \mathrm{C}\right)$. If not, there is likely a problem with the ground circuit or voltage supply. Check for 5 volts reference voltage at the connector. Also check for ground presence at the connector. If you do not have $5 \mathrm{~V}$ reference and/or ground continuity, check for these back at the PCM connector. If you have these at the PCM connector, then repair open or short between the PCM and the sensor. If you do not, 
remove the offending wire from the $\mathrm{PCM}$ and then check for proper voltage at the PCM pin. If it's present now, repair short on the circuit. If it is not present after removing the wire and checking the pin, replace PCM.

P1651: Ford - Power Steering Pressure Switch Input Malfunction

Possible causes

$>$ Vehicle towed with engine running

$>$ Faulty Power Steering Pressure (PSP)

$>$ Power Steering Pressure (PSP) harness is open or shorted

$>$ Power Steering Pressure (PSP) circuit poor electrical connection

$>$ PCM damaged

\section{Possible symptoms}

- Engine Light ON (or Service Engine Soon Warning Light) P0080: Exhaust Valve Control Solenoid Circuit High B1

This code is a generic OBD-II powertrain code, which means it applies to all makes and models of vehicles (1996 MY-till date), although specific repair steps may vary depending on the model. On vehicles equipped with variable valve timing (VVT), the Engine Control Module/Powertrain Control Module (ECM/PCM) controls the camshaft position by regulating the engine oil through the control solenoid to change the position of the camshaft. The control solenoid is commanded using a Pulse Width Modulated signal (PWM) from the ECM/PCM. The ECM/PCM monitors this signal and if the voltage is above specification, it will set this trouble code and illuminate the Malfunction Indicator Lamp (MIL). Bank 1 refers to the side of the engine with cylinder number 1 . The exhaust valve control solenoid is usually located on the Exhaust manifold side of the cylinder head. This code is similar to codes P0078 and P0079. This code may also be accompanied by $\mathrm{P} 0027$.

\section{Symptoms}

Symptoms of a P0080 may include: Check engine light illuminated (Malfunction indicator lamp) Vehicle may suffer from poor acceleration and decreased fuel economy.

Potential Causes

Potential causes may include:

$>$ Wire harness poor connections or disconnected

$>$ Control solenoid open circuit Short to power Faulty ECM

\section{Possible Solutions}

Wire harness - Check for unplugged harness connections, look for corrosion or loose wires to connectors. Unplug harness connectors from Solenoid and PCM, using a wiring diagram locate the + and - wires to the solenoid. The solenoid can be ground side or power side controlled, depending on the application. Check with factory wiring diagrams to determine the power flow in the circuit. Using a digital volt ohm meter (DVOM) set to the ohms setting, check for resistance between each end of the wire. An over limit reading on the DVOM may be an open in the wiring, loose connection or terminal. Control solenoid - With the electrical harness to the solenoid unplugged, using the DVOM set to ohms, check for resistance between each of the electrical terminals on the control solenoid itself. Use the factory specifications or a known good control solenoid if available to determine if there is resistance in the solenoid. If there is an over limit or very low resistance reading on the DVOM, the solenoid is likely faulty. Short to power - Unplug the harness to the PCM/ECM and locate the wires to the control solenoid. With the DVOM set to the volts scale, connect the negative lead to ground and the positive lead to the wire(s) to the control solenoid. Check for voltage, if there voltage present there may be a short to power in the wiring harness. Locate the short to power by unplugging harness connectors and testing the wiring back to the solenoid. PCM / ECM - If all wiring and the control solenoid checks out okay, it will be necessary to monitor the solenoid during engine operation by back probing the wires at the PCM/ECM. Using an advanced scan tool that will read the engine functions, monitor the duty cycle commanded to the control solenoid. It will be necessary to monitor the solenoid during engine operation under various engine RPM's and load. If the signal detected from the PCM is constantly on, there may be a fault with the PCM itself.

\section{P1451: Ford EVAP Control System Canister Vent Solenoid Circuit Malfunction}

This particular DTC article refers to a P1451 trouble code on Ford vehicles. This does not apply to other makes of vehicles. The EVAP system monitors the canister vent (CV) solenoid circuit for an electrical failure. The test fails when the signal moves outside the minimum or maximum allowable calibrated parameters for a specified canister vent duty cycle by PCM command.

Symptoms

You will likely not notice any drivability problems.

\section{Causes}

A code P1451 may mean that one or more of the following has happened:

$>$ VPWR circuit open

$>$ Damaged canister vent solenoid

$>$ Canister vent solenoid circuit open or shorted to ground, power, etc.

$>$ Damaged Powertrain Control Module (PCM)

\section{Possible Solutions}

$>$ Verify canister vent solenoid - To verify normal functioning, monitor the EVAP canister vent solenoid signal PID EVAPCV and the signal voltage (PCM control side). With the valve open, EVAPCV indicates $0 \%$ duty cycle and a voltage approximately equal to battery voltage. When the valve is commanded fully closed, EVAPCV indicates $100 \%$ duty cycle, and a minimum voltage drop of 4 volts is normal. Output test mode may be used to switch output on/off to verify function.

$>$ Replace canister vent solenoid

P1650 Ford - Power Steering Pressure Switch Out of SelfTest Range

In the key on engine off (KOEO) self-test, this DTC indicates the PSP input to the powertrain control module (PCM) is high. 
In the key on engine running (KOER) self-test, this DTC indicates the PSP input did not change state.

Possible causes

$>$ The steering wheel must be turned during key on engine off self-test

$>$ PSP switch/shorting bar damaged

$>$ PSP circuit open or shorted

\section{P0506 - Idle Air Control (IAC) System RPM Lower Than Expected}

This P0506 code is one that's sometimes triggered on vehicles that have electronic throttle control. That is, they don't have a regular throttle cable from the accelerator pedal to the engine. They rely on sensors and electronics to control the throttle. In this case, the P0506 DTC (diagnostic trouble code) is triggered when the PCM (powertrain control module) detects an engine idle speed that is lower than the desired (preprogrammed) RPM.

\section{Potential Symptoms}

Most likely the only thing that will be noticed is, the idle speed is lower than normal so it will likely be rougher. There are potentially other symptoms as well. Of course, when trouble codes are set, the MIL (malfunction indicator lamp, or the check engine light) will illuminates.

\section{Causes}

A P0506 DTC trouble code may be caused by one or more of the following:

$>$ A vacuum leak

$>$ An air restriction in the intake air path or exhaust

$>$ A faulty positive crankcase ventilation (PCV) valve

$>$ Damaged/failed/dirty throttle body

$>$ Internal engine problem

$>$ Failed PCM

\section{Possible Solutions}

This DTC is more of an informational code, so if there are any other codes set, they should diagnosed first. If there are no other codes, inspect for vacuum leaks, restrictions and damage. If there are no symptoms other than the DTC itself, just erase the code and see whether it returns.

\section{P0171: Lean Code for Cylinder Bank 1}

A General Motors/Ford P0171 is a LEAN code for cylinder bank 1, and P0174 is a LEAN code for cylinder bank 2 . These codes commonly occur on many GM/Ford vehicles, and are set when the powertrain control module (PCM) sees the airfuel mixture is running too lean (too much air, not enough fuel). When the Check Engine Light comes on, either one of these codes, or both, may be found when a code reader or scan tool is plugged into the vehicle diagnostic connector. A lean fuel condition may exist if the engine is sucking in too much air and/or the fuel system is not delivering enough fuel. If bad enough, a lean fuel condition may cause lean misfire, a rough idle, hesitation or stumble when accelerating, and/or poor engine performance. Unmetered air can enter the engine through a vacuum leak, a dirty airflow sensor that is not reading airflow accurately, an EGR valve is not closing and is leaking exhaust into the intake manifold, an EGR valve that is allowing too much flow (because the EGR differential pressure sensor that monitors EGR flow is faulty and is underreporting EGR flow).

\section{Possible Causes}

If the problem is not enough fuel, the underlying cause may be;

$>$ a weak fuel pump,

$>$ restricted fuel filter,

$>$ leaky fuel pressure regulator or

$>$ dirty fuel injectors.

$>$ dirty MAF sensor

$>$ vacuum leaks

$>$ low fuel volume delivery

\section{P1860: Chevrolet - TCC PWM Solenoid Circuit Electrical}

The Torque Converter Clutch Pulse Width Modulation (TCC PWM) solenoid valve controls the fluid acting on the converter clutch valve. The converter clutch valve controls the TCC application and release. The solenoid attaches to the control valve body within the transmission. The solenoid receives ignition voltage through circuit 239. The Powertrain Control Module (PCM) controls the solenoid by providing a ground path on circuit 418. Current flows through the solenoid coil according to the duty cycle (percentage of $\mathrm{ON}$ and $\mathrm{OFF}$ time). The TCC PWM solenoid valve provides a smooth engagement of the torque converter clutch by operating during a duty cycle percent of $\mathrm{ON}$ time.

\section{Possible causes}

$>$ Faulty Torque Converter Clutch Pulse Width Modulation (TCC PWM) Solenoid

$>$ Torque Converter Clutch Pulse Width Modulation (TCC PWM) Solenoid harness is open or shorted

$>$ Torque Converter Clutch Pulse Width Modulation (TCC PWM) Solenoid circuit poor electrical connection.

When the PCM detects a continuous open, short to ground or short to power in the TCC PWM solenoid valve circuit, then DTC P1860 sets. DTC P1860 is a type B DTC.

\section{Possible symptoms}

Engine Light ON (or Service Engine Soon Warning Light)

Analysis of DTCs from Selected Japanese Vehicles

P0500: Vehicle Speed Sensor Malfunction

Basically this means that the vehicle's speed as read by the Vehicle Speed Sensor (VSS) is not working properly.

\section{Symptoms}

Symptoms of a P0500 DTC may include:

$>$ loss of anti-lock brakes

$>$ the "anti-lock" or "brake" warning lamps on the dash may be lit

$>$ the speedometer or odometer may not work properly (or at all)

$>$ the vehicle's RPM limiter may be decreased the shifting of an automatic transmission may become erratic other symptoms may also be present 


\section{Causes}

A code P0500 may mean that one or more of the following has happened:

$>$ The Vehicle Speed Sensor (VSS) is not reading (functioning) properly

$>$ There is a broken/frayed wire leading to the vehicle speed sensor

$>$ The vehicle's PCM is not correctly configured for the actual tire size on the vehicle

\section{Possible Solutions}

The simplest thing to do is to reset the code and see if it comes back. Next, check all wiring and connectors that lead to the speed sensor. The location of the sensor depends on your vehicle. The sensor could be on the rear axle, transmission, or perhaps the wheel hub (brake) assembly. If the wiring and connectors are $\mathrm{OK}$, then check the voltage at the speed sensor. Again, the exact procedure will depend on your make and model of vehicle. If everything looks $\mathrm{OK}$, the sensor should be replaced.

\section{P1135: Heated Oxygen Sensor Heater Circuit Malfunction} The P1135 is set when the Engine Control Module (ECM) detects a heated oxygen sensor heater circuit malfunction. AirFuel Ratio (A/F) sensor need to reach a minimum operating temperature of $1200^{\circ} \mathrm{F}\left(649^{\circ} \mathrm{C}\right)$ to produce an accurate voltage signal. The faster the air-fuel ratio sensor reaches that temperature the faster the sensor will start sending an accurate signal to the Engine Control Module (ECM). In order to achieve the require temperature, a heater element is included inside the air-fuel ratio sensor. The ECM controls the air-fuel ratio sensor heater element based on signals from the engine coolant temperature and engine load. The ECM controls the heater element circuit by allowing current flow to ground. The ECM monitors the voltage signal received through the heater element circuit and determines the state of the circuit by comparing the voltage detected with the factory specifications.

\section{Possible causes}

$>$ Faulty Air-Fuel Ratio (A/F) Sensor Bank 1 Sensor 1

$>$ Air-Fuel Ratio (A/F) Sensor Bank 1 Sensor 1 harness is open or shorted

$>$ Air-Fuel Ratio (A/F) Sensor Bank 1 Sensor 1 circuit poor electrical connection

$>$ Faulty Engine Control Module (ECM)

\section{P0011 Nissan - Intake Valve Timing Control Performance Bank 1}

This mechanism hydraulically controls cam phases continuously with the fixed operating angle of the intake valve. The ECM receives signals such as crankshaft position, camshaft position, engine speed, and engine coolant temperature. Then, the ECM sends ON/OFF pulse duty signals to the intake valve timing control solenoid valve depending on driving status. This makes it possible to control the shut/open timing of the intake valve to increase engine torque in low/mid speed range and output in high-speed range. The intake valve timing control solenoid valve changes the oil amount and direction of flow through intake valve timing control unit or stops oil flow. The longer pulse width advances valve angle.
The shorter pulse width retards valve angle. When $\mathrm{ON}$ and OFF pulse widths become equal, the solenoid valve stops oil pressure flow to fix the intake valve angle at the control position. When there is a gap between angle of target and phase-control angle degree, the valve will stop working and the ECM will trigger the P0011 code.

Possible symptoms

$>$ Engine Light ON (or Service Engine Soon Warning Light)

$>$ Possible engine lack/loss of power

$>$ Possible engine rough idle

Possible causes

$>$ Faulty Intake Valve Timing Control Solenoid Valve

$>$ Intake Valve Timing Control Solenoid Valve harness is open or shorted

$>$ Intake Valve Timing Control Solenoid Valve circuit poor electrical connection

$>$ Faulty Camshaft Position Sensor

$>$ Camshaft Position Sensor harness is open or shorted

$>$ Camshaft Position Sensor circuit poor electrical connection

$>$ Faulty Crankshaft Position Sensor (POS)

\section{Possible Solutions}

This DTC code is a result of a mechanical fault of the VCT (variable camshaft timing) unit or related components, so there is no need for electrical diagnosis.

P0420: Catalyst System Efficiency below Threshold (Bank 1) This diagnostic trouble code (DTC) is a generic powertrain code. It is considered generic because it applies to all makes and models of vehicles (from 1996 MY-Till date), although specific repair steps may be slightly different depending on the model. So this engine code article applies to Nissan, Toyota, Ford, GM, etc. The catalytic converter is a part of the exhaust system that looks kind of like a muffler, although its job is very different from that of a muffler. A catalytic converter's job is to reduce emissions from the exhaust. The catalytic converter has an oxygen sensor in front and behind it. When the vehicle is warm and running in closed loop mode, the upstream oxygen sensor waveform reading should fluctuate. The downstream $\mathrm{O}_{2}$ sensor reading should be fairly steady. Typically the P0420 code triggers the Check Engine Light if the readings of the two sensors are similar. This is indicative of (among other things) a converter that is not working as efficiently as it should be (according to specifications). Catalytic converters are not normally a "wear" type item. That is they are not designed to wear out and need replacement. If they have failed, it is likely due to something else that caused it to fail.

\section{Symptoms}

The main symptom to the driver is the illumination of the MIL (malfunction indicator lamp). You will likely not notice any drivability problems, although there may be symptoms. For example, if the substance inside the catalytic converter is broken or failed, it may be restricting the exhaust which will result in a feeling of reduced power output from the vehicle. 


\section{Causes}

A code P0420 may mean that one or more of the following has happened:

$>$ Leaded fuel was used where unleaded was called for

$>$ A damaged or failed oxygen sensor $\left(\mathrm{H}^{O_{2} \mathrm{~S}}\right)$

$>$ Downstream oxygen sensor $\left(\mathrm{H}^{O_{2} \mathrm{~S}}\right)$ wiring damaged or connected improperly.

$>$ The engine coolant temperature sensor is not working properly

$>$ Damaged or leaking exhaust manifold / catalytic converter / muffler / exhaust pipe

$>$ Retarded spark timing

$>$ The oxygen sensors in front and behind the converter are reporting too similar of readings

$>$ Leaking fuel injector or high fuel pressure

$>$ Cylinder misfire

$>$ Oil contamination

\section{Possible Solutions}

Some suggested steps for troubleshooting a P0420 error code include:

$>$ Check for exhaust leaks at the manifold, pipes, catalytic converter. Repair as required.

$>$ Inspect the downstream heated oxygen sensor $\left(\mathrm{H}^{\mathrm{O}_{2} \mathrm{~S}}\right)$, replace if necessary

$>$ Replace the catalytic converter

The overall and probably the biggest mistake vehicles owners make when they have a P0420 code is to simply replace an oxygen sensor $\left(\mathrm{H}^{O_{2}} \mathrm{~S}\right)$. It is important to do proper diagnosis so you are not wasting money replacing parts unnecessarily.

\section{P0421: Warm Up Catalyst Efficiency below Threshold (Bank 1)}

Basically this means that the oxygen sensor downstream of the catalytic converter on bank 1 is detecting that the converter is not working as efficiently as it should be (according to specifications). It is part of the vehicle emissions system.

\section{Symptoms}

You will likely not notice any drivability problems, although there may be symptoms. The code may be more likely to occur after the engine has had numerous cold starts in the past 1-2 days.

\section{Causes}

A code P0421 may mean that one or more of the following has happened:

$>$ The catalytic converter is no longer functioning properly

$>$ An oxygen sensor is not reading (functioning) properly

$>$ A spark plug is fouled up

\section{Possible Solutions}

Measure the voltage at the oxygen sensor on Bank 1 (the rear sensor, or the sensor after the converter). In fact, it would be a good idea to test each oxygen $\mathrm{O}_{2}$ sensor while you're at it.

\section{P0304 Code - Cylinder Number 4 Misfire Detected}

A P0304 code means that the vehicle's computer has detected that one of the engine's cylinders is not firing properly. In this case it is cylinder Number 4.

\section{Symptoms}

Symptoms may include:

$>$ the engine may be harder to start

$>$ the engine may stumble, and/or hesitate

$>$ other symptoms may also be present like illumination of the MIL

\section{Causes}

A code P0304 may mean that one or more of the following has happened:

$>$ Faulty spark plug or wire

$>$ Faulty coil (pack)

$>$ Faulty oxygen sensor(s)

$>$ Faulty fuel injector Burned exhaust valve

$>$ Faulty catalytic converter(s)

$>$ Running out of fuel Poor compression

\section{Possible Solutions}

If there are no symptoms, the simplest thing to do is to reset the code and see if it comes back. If there are symptoms such as the engine is stumbling or hesitating, check all wiring and connectors that lead to the cylinders (i.e. spark plugs). Depending on how long the ignition components have been in the car, it may be a good idea to replace them as part of the regular maintenance schedule. It is also suggested that spark plugs, spark plug wires, distributor cap, and rotor (if applicable) be replaced. Otherwise, check the coils (coil packs). In some cases, the catalytic converter has gone bad. If there is smell of rotten eggs in the exhaust, then the cat converter needs to be replaced. In other cases the problems may also be faulty fuel injectors.

\section{P0135: Oxygen $\mathrm{O}_{2}$ Sensor Heater Circuit Malfunction (Bank 1, Sensor 1)}

This code refers to the front oxygen sensor on Bank 1. The heated circuit in the oxygen sensor decreases time needed to enter closed loop. As the $\mathrm{O}_{2}$ heater reaches operating temperature, the oxygen sensor responds by switching according to oxygen content of the exhaust surrounding it. The ECM tracks how long it takes for the oxygen sensor to begin switching. If the ECM determines (based on coolant temp) that too much time elapsed before the oxygen sensor began operating properly, it will set P0135.

Symptoms

It will likely be noticed that poor fuel economy prompt the illumination of the MIL.

\section{Causes}

A code P0135 may mean that one or more of the following has happened:

$>\mathrm{O}_{2}$ Heater element resistance is high

$>$ Internal short or open in the heater element

$>\mathrm{O}_{2}$ heater circuit wiring high resistance

$>$ open or short to ground in the wiring harness 
Possible Solutions

$>$ Repair short or open or high resistance in wiring harness or harness connectors

Replace oxygen sensor (cannot repair open or short that occurs internally to sensor)

\section{CONCLUSION}

From the research carried out and the various results obtained from the various diagnostics of some selected automobiles from German Make, American Make, and Japanese Make vehicles, the diagnostics results provided an appreciated feedback through the triggering of Malfunction Indicator Light (MIL) and thereby with the use of diagnostic scan tools, the failures and faults within the engine compartment were detected. It was evident that On Board Diagnostic has the capacity to facilitate and enhance the early detection of vehicle malfunction and faults related to emissions control components and as a result reducing high emissions caused by emission related malfunctions. Intelligent analysis of the resulted diagnostic trouble codes provided grounds for better understanding of present diagnostic systems which thus poses the weakness of the present On Board Diagnostics inability to detect a prevailing failure and fault in a situation where the malfunction indictor light (MIL) fail to trigger $\mathrm{ON}$.

\section{ACKNOWLEDGMENT}

Firstly, I want to thank Almighty God for His Providence and wisdom, knowledge and understanding. I wish to express my heartfelt gratitude to my Project Supervisor Engr. Professor M.S. Abolarin for providing me with guidance and support throughout the evolution of this project, as well as the Mechanical Engineering Department of FUT, Minna for providing me with all the necessary resources and knowledge to complete this research project. For the important role and contribution of my Case Study Workshop for the materials and various data collected for this research I appreciate the Director of Sarki Pawa Automobile Workshop, Minna and also all the technicians at the workshop especially Mallam Zakare

\section{REFERENCES}

[1] J. Erjavec, Automotive Technology, 4th ed. New York: Thomson Delmar Learning, 2005.

[2] B. Hollembeak,. Classroom Manual for Automotive Fuels \& Emissions. New York: Thomson Delmar Learning, 2005.

[3] Society of Automotive Engineer. On-Board Diagnostics for Light and Medium Duty Vehicles Standards Manual, ( 2003) ed. SAE International: USA. 